\title{
EDITORIAL
}

\section{The future of pain}

Unrelieved pain has major negative effects on the lives of millions of people worldwide, and is associated with huge societal costs. Breakthroughs in pain therapy have been rare in the past 50 years, but there is increasing hope that advances in multiple areas of pain research could lead to the introduction of much-needed novel and more effective analgesics.

The traditional theory of pain, which evolved during the early twentieth century, holds that pain is a specific sensation produced by a direct-line pain pathway from pain receptors in the body to a pain centre in the brain. Research based on this theory has investigated physiological mechanisms at every level of the pathway, from receptors to cerebral cortex, and has produced a variety of drugs to control acute pain due to injury or disease. However, it has not yet led to any major new drugs to control chronic pains such as the neuralgias and phantom limb pain, which represent a vast unmet medical need. It therefore seems timely to reflect on how pain research has evolved in the past five decades, and consider where therapeutic breakthroughs in this field might stem from in the future.

The gate control theory of pain, proposed in 1965, focused on chronic pain and postulated that neural gates in the spinal cord can be opened or closed by signals descending from the brain, as well as by sensory information ascending from the body. The theory also highlighted the psychological functions of the brain and produced an explosive growth of knowledge related to pain. Brain research, especially in neuropharmacology and cognitive neuroscience, has led to the development of new antidepressant and anticonvulsant drugs, as well as several psychological techniques that produce substantial relief from chronic pain. The gate control theory also stimulated a shift away from the traditional direct-line pain pathway to the concept of parallel neural networks in the brain associated with the sensory, affective and cognitive dimensions of pain ${ }^{1}$. By doing so, it connects the field of pain to exciting current research on the mechanisms in the brain that generate the experience of pain.

For example, phantom limb pain occuring after the amputation of an arm or leg presents a huge challenge to pain theory and research. The pain is intense and persists for years after the stump has healed. A high-level cordectomy - total removal of several segments of spinal cord so that sensory information from the pelvis and legs is unable to arrive at the brain - does not stop intense pain in the phantom part of the body. Even anaesthetic blocks of the sympathetic ganglia above the cordectomy fail to stop the pain. The extraordinary reality of painful phantom limbs indicates that the brain does more than detect and analyse sensory inputs; it generates the perceptual experience of limbs and pain in the absence of external inputs.

Such findings help to illustrate the extent of the challenges in pain research. However, now, at the beginning of the twenty-first century, there are several reasons to be optimistic about the future of pain research and therapy. First, imaging techniques are increasingly being used to study physiological events in the brain of human beings while they simultaneously report their subjective pain experiences. These techniques have confirmed painrelated activity in widely distributed, highly interconnected areas in the brain, and support the theory that pain is generated by a complex neural network ${ }^{1}-$ the "body-self neuromatrix" - which has multiple inputs and outputs. The theory expands the scope of pain research to include the brain mechanisms that integrate these widespread neural activities into united streams of awareness, as well as the neural and endocrine programmes that regulate stress associated with injury and disease.

Second, the detailed knowledge and technical skills developed for research on the spinal cord can be used to explore brain mechanisms in humans and animals. The shift to a top-down strategy that begins with brain function and conscious experience will expand the field of pain research by incorporating the rapidly growing knowledge of cognitive neuroscience and the evolution of the brain. The potential of this "hybrid vigour" is a direct attack on pain and suffering in the structures that generate them.

Third, our knowledge of the genetic basis of the development of the brain is growing rapidly and, at the same time, a large number of genes related to pain have been identified. The study of pain, therefore, has broadened and now incorporates research in epidemiology and medical genetics as well as sociological and cultural studies. The inevitable convergence of these three approaches to reveal the functions of the brain will hopefully lead to the relief of the pain and suffering that is currently endured by millions of people.

Ronald Melzack

. Melzack, R. Evolution of the neuromatrix theory of pain. Pain Pract. 5, 85-94 (2005). 